\title{
LA OBRA BOTÁNICA DE ANTONIO PARRA
}

\section{Armando García González}

\section{RESUMEN}

Nuestro objetivo es estudiar la labor realizada por el naturalista cubano Antonio Parra entre 1790 y 1792, que no se limitó a la confección de herbarios y al envío de plantas y semillas a la metropoli con el fin de aclimatarlas a la península, sino que profundizó en la redacción de un catálogo y un discurso sobre los medios de connaturalizar en España los árboles de la Habana.

\section{SUMMARY}

Our goal is study the work carried out by the Cuban naturalist Antonio Parra between 1790-1792, that didn't limit to the making of herbariums and to the shipment of plants and seeds to Spain with the purpose of acclimatizing them to the Peninsula, but rather he fathomed in the composition of a catalog and a discourse on the means of naturalize in Spain the trees of the Habana.

Es evidente que el movimiento cultural al que se ha dado el nombre de ilustración favoreció el desarrollo de las ciencias y las artes no sólo en los países europeos, sino también en las colonias que éstos tenían en 
América, donde ejerció una influencia verdaderamente positiva e impulsora. Dicho proceso no puede ni debe reducirse, sin embargo, a una mera influencia. Fue más bien un fenómeno complejo en que intervinieron factores sociales, políticos y económicos que matizaban y por lógica razón diferenciaban los intereses de unas y otras regiones, en medio de convulsos cambios, adaptaciones y readaptaciones provocados por las políticas seguidas en esos países. En el caso de Cuba esto es palmario. Acorde con el desarrollo alcanzado por la isla, y sobre todo por su parte occidental, entre otras cosas gracias al avance de la agricultura cañera, consolidada mediante la esclavitud, se había constituido en la segunda mitad del siglo XVIII una élite, representada por grandes hacendados y comerciantes. Asimismo era posible hallar médicos, filósofos, pedagogos, que conformaban una clase media, formada en instituciones francesas y españolas o en la propia isla, donde ya eran conocidas la Real y Pontificia Universidad de la Habana y el Seminario de San Carlos. Así, tanto en el plano intelectual, como político y económico, se dejaban ver ya las contradicciones entre los intereses de esta, si se quiere burguesía de la isla, y la de la península, y por ese motivo el inicio de una conciencia nacional que adquirió aquélla, con mayor definición, en la centuria siguiente.

Cuando Antonio Parra y Callado arriba a Cuba en 1763 tal situación no es tan subrayada. Tras un año de dominación inglesa en la isla, España recibía ésta a cambio de la Florida, luego del Tratado de Versalles. A Cuba llegaba, con el conde de Ricla, Ambrosio Funes de Villalpando, una fuerte tropa, constituida por un gran tren de artillería, cuatro navíos de guerra y unos dos mil hombres ${ }^{1}$, entre los que figuraba Parra probablemente. Portugués de nacimiento - había nacido en Tavira, antiguo reino de los Algarves, el 27 de junio de 1739- vino a La Habana como soldado del regimiento de Infantería de Mallorca. Pero pronto se licenció para contraer matrimonio en 1765 ó 1766 con Catarina Gertrudis Muñoz, de

1 Jimeno, F. (1881): «Efemérides cubanas». Revista de Cuba. La Habana, t. 12, pp. 31 44; p. 31

2 Las partidas de bautismo de los hijos de Parra, así como de su segundo matrimonio las reproduce Pérez BeATo, M. (1909): «Don Antonio Parra; documentos referentes a este naturalista y a su hijo el dibujante y grabador cubano Manuel Antonio Parra». «El Curioso Americano». La Habana. Año 2, n. ${ }^{\circ} 11$ y 12, pp. 18-25, enero-febrero; Año 3, n. ${ }^{\circ}$ 2, pp. 41-48, marzo-abril. 


\section{LA OBRA BOTÁNICA DE ANTONIO PARRA}

la cual tuvo varios hijos ${ }^{2}$, todos nacidos en Cuba. Viudo de su primera esposa, contrajo nuevas nupcias en 1784 con María Blaza del Río.

Las aficiones naturalistas de Parra se estimularon, desde su llegada, ante la hermosura de la isla y su gran variedad de plantas y animales, de manera que dedicaba los ratos libres que le permitían sus obligaciones como militar a conformar una colección de "producciones marinas". Comenzó primero por éstas, recolectando conchas, estrellas de mar, etcétera, y a partir de 1776 se dedicó además a disecar peces, mediante un método - que él llamó de su invención- de vaciarlos totalmente, aplicar alguna sustancia que los endurecía y darles color, a fin de hacerlos más «reales» y atractivos.

Parra no sólo colectó y preparó peces, crustáceos, reptiles, equinodermos, celenterados y espongiarios, sino que tuvo asimismo animales vivos, como caimanes, cocodrilos y monos. Este gabinete, que formó en su casa de La Habana intramuros (Tejadillo n. ${ }^{\circ} 8$ ), estuvo abierto al público y constituyó uno de los acontecimientos culturales más llamativos de su época en dicha capital. Le visitaron figuras nacionales y extranjeras, reflejándose su interés en la prensa citadina de entonces.

A modo de una especie de catálogo con que presentar sus colecciones, Parra preparó y dio a la imprenta, lo que hoy se considera el primer libro científico publicado en Cuba, Descripción de diferentes piezas de historia natural, las más del ramo marítimo, que vio la luz en 1787, y contaba con setenta y cinco láminas grabadas en cobre por su hijo Manuel Antonio cuando sólo tenía dieciséis años. El propio Manuel Antonio iluminó, es decir, dio color a los grabados del libro, que al parecer se editó dos veces ${ }^{3}$.

La labor de Parra estuvo precedida hasta cierto punto por las reales órdenes que llegaban a las colonias para que acopiasen animales, minerales, plantas y objetos curiosos que pudieran ser incorporados a los Reales Gabinete de Historia Natural y Jardín Botánico de Madrid. En ese sentido, mantuvo correspondencia con figuras relacionadas con estas instituciones y en general con el gobierno metropolitano. Toda su colección la vendió al Gabinete en 1788 ó 1789 y se la entregó, enriquecida, cuatro años después. Obtuvo por ello cuatro mil pesos como pago de sus gastos, y además una pensión de otros dos mil, pagaderos por las reales Cajas de La Habana.

\footnotetext{
${ }^{3}$ Para estos y otros datos ver García González, A. (1989): Antonio Parra en la ciencia hispanoamericana del siglo XVIII. La Habana. Editorial Academia.
} 


\section{ARMANDO GARCíA GONZÁLEZ}

\section{Un proyecto botánico}

Durante esos años en que preparaba sus colecciones, Parra se interesó además por la botánica. Era imposible escapar a todo el movimiento que en torno a esa disciplina se producía en diversos países civilizados, entre ellos España. Las ideas linneanas comenzaban su empuje para desplazar las de Tournefort y otros botánicos que habían primado por entonces. La recolección de plantas, su herborización y clasificación motivaba desde años atrás a muchos investigadores, médicos y sabios, no sólo por ansia de saber, sino también preocupados por conocer las múltiples aplicaciones de los vegetales, desde puntos de vista comerciales, industriales, medicinales y ornamentales.

El descubrimiento de América había mostrado a los europeos un mundo animal y vegetal hasta entonces desconocido, pero exhuberante y hermoso, donde podían hacerse, como en efecto se hicieron, importantes descubrimientos. En el siglo XVIII, la mayor parte de ese mundo biológico aún permanecía en gran medida desconocido para Europa. Hasta esa fecha la historia natural de Cuba se difundió fundamentalmente a través de visitas foráneas o de los propios colonizadores españoles. Estos últimos recogieron desde sus inicios, en crónicas y diarios de viaje, las primeras noticias acerca de la flora y fauna cubanas, ampliamente divulgadas en los siglos siguientes, mediante compendios, casi siempre geográficos y botánicos, que publicaron los exploradores que recorrieron la isla, y entre los que se encontraban alemanes, franceses, ingleses, suecos, húngaros y de otras nacionalidades. Uno de ellos, Federico W. Nascher, escribió la primera obra sobre la flora de Cuba, Flora cubana, exhibens generum especierum circa Havana crescentium, publicada en Leipzig en 1758. Coincidiendo con Parra, arribó en el propio año de 1763 el naturalista C. A. Wallerton para recolectar ejemplares y escribir un Traité explicatif d' un herbier, que se publicó entre 1767 y 1770, y contenía nombres de plantas medicinales de Cuba.

Por otra parte, desde 1777 hasta fines del siglo XVIII se organizan y envían desde España varias expediciones científicas, de las que podemos mencionar las efectuadas a los reinos del Perú y Chile (1777-1787), al Nuevo Reino de Granada (1783), a Nueva España (1787) y la de Alejandro Malaspina alrededor del mundo (1789). Estas expediciones tenían entre otras misiones las de estudiar la flora y fauna de los distintos países visitados, recolectando semillas y plantas (vivas o herborizadas) y animales. En el caso de las primeras consignaban sus usos industriales, eco- 
nómicos, medicinales, artísticos $\mathrm{u}$ ornamentales. Ello conllevaba, por supuesto, la posibilidad de aclimatarlas en la península, política seguida por los reyes Carlos III y Carlos IV, al encomendar dicha tarea al Real Jardín Botánico de Madrid, desde su fundación en 1781. También se crearon jardines de aclimatación en distintas regiones de España, como en Aranjuez, Valencia y Cartagena, donde se prepararon condiciones para recibir, sembrar temporalmente y trasladar a su destino definitivo aquellas plántulas y semillas importantes, de acuerdo con los usos y utilidades mencionados.

Como recibiera Parra en Madrid encargo de que remitiese a España todos las plantas que creyese conveniente, decidió realizar esta labor en relación con los árboles que se cultivaban en Cuba, a fin de que se hiciese otro tanto en el Jardín Botánico de Madrid y en los jardines de aclimatación de Aranjuez. En efecto, en 1790 el portugués envió en la fragata Rosario y en las urcas Polonia y Redentora, ocho cajones con plantas sembradas en canutos de barro ${ }^{4}$, de mameyes colorados (Calocarpon) y de Santo Domingo (Mammea), zapotes (Achras, Pouteria, Dyospiros), anones (Annona), cedros (Cedrela), ceibas (Ceiba) en arbolitos y semillas, almácigos (Bursera), guayabos del Pinar y del Perú (Psidium) y palmas reales (Roystonea). Acompañaba su envío con noticias acerca de las utilidades y descripciones de dichas plantas, así como algunas instrucciones de cómo sacarlas de los cajones y el modo de conservarlas.

Al respecto de esto último decía, por ejemplo: «Cada cajón lleva ocho arbolitos, cada uno de ellos plantado en un caño de barro de media vara de largo y seis pulgadas de diámetro, abierto por uno y otro lado para que las raíces que más crecieren, salgan por abaxo y puedan extenderse en un suelo de tierra en que van sentados dichos caños. Este método me ha parecido el más seguro para transportar los arbolitos para que al tiempo que se trasplanten no se lastimen sus raíces. El modo de sacar los arbolitos es romperle un frente, y se descubran los caños, luego con facilidad se tira para fuera teniendo la precaución de ponerles la mano debaxo o una tablita para sostener la tierra y de ese modo se colocan en el

\footnotetext{
${ }^{4}$ Estos envíos motivaron una determinada correspondencia, algunas de cuyas misivas reproduce Barras de Aragón, de quien tomamos varios de estos datos. Véase BARRAS De ARAGón, F. (1950): «Don Antonio Parra como botánico». Boletín de la Real Sociedad de Historia Natural de Madrid. t. XLVIII, pp. 219-244. Las cartas en cuestión se encuentran en el Archivo de Indias. Fondo Indiferente General. Legajo 1546.
} 
hoyo, que deberá estar abierto, y sentado el caño, se rompe dentro del hoyo, regándolo antes para que la tierra quede unida al sacar los tiestos y se terraplena; pues de este modo no hará ningún sentimiento la planta» ${ }^{5}$.

Ese mismo año despachó con parecidas indicaciones doce cajones más en los buques Asia y Castilla, conteniendo esta vez cedros, cocos (Cocos), palmas reales, guacamayas (Poinciana, Cassia), guásimas (Guasuma), anones, guiras (Crescentia), mameyes, atejes (Cordia), caimitos (Chrysophylum), mamoncillos, guanábanas (Annona), guayabos del Pinar y del Perú, tamarindos (Tamarindus), zapotes y aguacates (Persea). En 1791 y 1792 hizo Parra nuevos envíos, añadiendo además de estas plantas otras de piñones (Jatropha, Erythrina), uveros (Coccoloba), lirio francés o castaños (Pristimera), cerillos (Exostema), cañas bravas (Bambusa) y papayas (Carica). También aquí informaba acerca de la utilidad comercial, medicinal e industrial que brindaba cada una de ellas, la descripción de sus flores y frutos y otros detalles que consideró de interés.

Si bien las descripciones de Parra ofrecen poco interés desde el punto de vista botánico, no resultan tan así desde el utilitario; único objetivo que tiene en mente el portugués al hacer sus envíos. Así, al hablar de las utilidades alimenticias y medicinales de algunas plantas dice, por ejemplo, que el coco da un agua medicinal, fresca y agradable para beber, y su masa también sirve de alimento: tanto de una como de la otra "se hace un gran consumo, sacan leche y manteca para cocinar empleando gran cantidad en dulces en almíbar, seco, tortas y otros usos" ${ }^{6}$.

Las palmas reales tienen diversos usos, y producen además un fruto o palmiche, del cual se extrae una masa blanca, insípida, compacta y muy recia, que contiene mucho aceite. El fruto del tamarindo es comestible (en refrescos y dulces) y de uso bien conocido en medicina; el de los piñones es un admirable purgante y el del guayabo del Perú es dulce, de olor agradable y buen gusto; la raíz es utilizada por la gente de campo como astringente. Las hojas, flores y frutos de la guira chica son medicinales; también lo son las hojas del almácigo, etcétera.

${ }^{5}$ BARRAS, op. cit., pp. 225 y 227.

6 Ibidem, p. 227. 


\section{LA OBRA BOTÁNICA DE ANTONIO PARRA}

En relación con los usos industriales, expresa que la madera de esta última planta, como la de la guira grande, es blanca y de larga duración y resistencia, empleándose en ejes de carrozas y otros usos. La del manzanillo, fuerte y de color pajizo, se utiliza para confeccionar distintos utensilios en el campo. Lo mismo ocurre con la del zapote culebra y la del mamoncillo. La del aguacate es blanca y floja utilizándose en la construcción de cajas para el azúcar, y la de la guásima, también blanca y suave, en hormas de zapatos. Por su parte, la del cerillo, amarilla, sólida y de buen lustre, se emplea en enchapados y obras de tornería. Igualmente hace referencia a frutos que son utilizados para pastos de animales, maderas empleadas en la confección de cercas, y arbustos que podían servir de ornamentación en jardines.

Algunos datos tienen particular interés pues muestran cómo se consumían por la población distintos alimentos y frutos, hábitos alimentarios prácticamente desaparecidos en la actualidad. Por ejemplo, al referirse al mamey de Santo Domingo, dice que se comía en tajadas y cubiertas de vino y canela, como postres. De la médula de la semilla del aguacate, cocida y desaguada, se confeccionaban turrones y otros: La introducción de nuevos alimentos, incluso de otras culturas alimentarias, pero también los estudios posteriores sobre la toxicidad y en general trastornos en el organismo de ciertos componentes utilizados en la alimentación debieron ser algunos de los factores que determinaron estos cambios.

En 1791 Parra mandó doce cajones más de plantas vivas en los buques Asia y Castilla. Las mismas llegaron bien, trasladándose a los jardines del Puerto de Santa María, hasta que pudieran remitirse luego a Aranjuez. El 11 de agosto de 1792 envía cuatro cajones en la fragata Santa Balbina y la urca Santa Rita, y el 19 de mismo mes otras cuatro en las naves Espaciosa y Anunciación ${ }^{7}$, todas con el mismo destino.

La actividad recolectora del portugués, más los largos años que había vivido en la isla, le permitieron adquirir conocimientos que en cierta medida reflejó en sus remisiones. En 1799 publicó en Madrid un Discurso sobre los medios de connaturalizar en España, los cedros de la Havana, y otros árboles, así como de construcción, como de maderas curiosas y frutales. En este folleto - que además permite conocer la suerte corrida por algunas de las plantas que envió- se abordaron las recomendaciones

\footnotetext{
7 Ibidem.
} 
para trasplantar y connaturalizar en la península los diferentes árboles mencionados, aprovechables en la agricultura, las fábricas y la cría del ganado.

Su idea básica consistía en crear parajes adecuados para el depósito de arbolitos hasta que pudieran trasplantarse a un terreno apropiado. Por eso recomendaba que en las inmediaciones del puerto de Cádiz —el más frecuentado por las embarcaciones que llegaban de América- se construyeran "criaderos de plantas" y semilleros, sirviendo este lugar además como depósito de las que venían con destino a los reales jardines botánicos, pues en ocasiones llegaban maltratadas por el descuido o la larga travesía. En ese lugar se repondrían hasta que estuvieran en disposición de ser remitidas a su destino.

En Andalucía y otras tierras las personas aficionadas y amantes del progreso, así como diferentes personalidades (curas, párrocos, alcaldes) e instituciones (Sociedades Económicas, etcétera) podían encargarse de recoger, transportar y sembrar plántulas, según fueran llegando las remesas. Todo ello bajo el control de un Comisionado que mantendría correspondencia con el Jardín Botánico de Madrid para conocer la marcha de estos trabajos.

Parra insistió en la importancia de los árboles maderables y sobre todo de los cedros, pues estimaba que prevalecerían en España, ya que prosperaban en todos los terrenos de Cuba, y además porque de los cuarenta y cinco que había enviado entre 1790 y 1792, y se hallaban en el Real Jardín de Aranjuez, algunos habían "prendido" bajo determinadas condiciones (en estufas), aunque podían darse bien al aire libre. En ese sentido especificó todos los cuidados que debían tenerse en cuenta para lograrlos.

Los envíos de plantas efectuados por Parra desde La Habana arribaron al Puerto de Santa María, atendido por Pedro Gutiérrez, y desde allí se trasladaron a los jardines de Aranjuez, al cuidado del Jardinero Mayor Pablo Bortelou. Estos envíos no sólo parecen encontrarse entre los primeros que en gran escala se mandaron a la península durante el siglo XVIII, sino también un factor importante es el hecho de que algunas plantas remitidas por Parra pudieron ser introducidas por vez primera en España -o al menos no tenemos referencias anteriores-, como son el ateje (Cordia cocolloca), el mamoncillo (Mellicoca bijuga), el cedro (Cedrela mexicana), el piñón espinoso (Erithrina cubensis), el almácigo (Bursera simaruba), la palma real (Roystonea regia), el abrojo de la Florida (Peireskia cubensis), el anón (Annona squamosa) y otras. Algunas de las plan- 


\section{LA OBRA BOTÁNICA DE ANTONIO PARRA}

tas remitidas por Parra se sembraron en el Jardín Botánico de Madrid, según revelan los catálogos de siembra, y otras aún se cultivan en España ${ }^{8}$.

Aunque el folleto de Parra no constituye un trabajo netamente científico, pues sólo consigna los nombres vulgares de las plantas cubanas (más de trescientas) y no los científicos, sino que más bien tiene como finalidad una utilidad práctica, la labor de este naturalista puede considerarse dentro de los antecedentes significativos de la botánica en Cuba. A su partida, en el propio año de 1793, se funda en la isla la Sociedad Económica de Amigos del País de La Habana, la cual proclamó entre sus primeras tareas la creación de un Jardín Botánico, así como de una escuela de química con su laboratorio. Todo ello, a fin de facilitar la instrucción, pues le interesaba el desarrollo de esas ciencias por su directa aplicación a la producción azucarera, objetivo fundamental de los hacendados criollos. Pero, como se sabe, no sería hasta el siglo XIX cuando se verían cristalizados esos deseos. Entre los promotores que abogaron por el avance de la botánica se encontraban, además, Mariano Espinosa y Pedro Le Compte, dos figuras que pertenecieron a dicha Sociedad y que, como Parra, enviaron a España muchas muestras de plantas cubanas para ser allí aclimatadas y connaturalizadas.

\footnotetext{
8 Estos datos los recoge CAMPo SERRANO, I. (1993): Introducción de plantas americanas en España. Madrid. Ministerio de Agricultura, Pesca y Alimentación.
} 


\begin{abstract}
APENDICE 1
A continuación ofrecemos una lista con la clasificación de las plantas que Parra envió, y para lo cual hemos utilizado el Diccionario Botánico de Nombres Vulgares Cubanos, de Juan Tomás Roig. 4. ${ }^{\text {a }}$ ed. La Habana, 1975.

Abrojo de la Florida. Peireskia cubensis, Britton y Rose (cactáceas).

Aguacate. Persea americana, Mill. (lauráceas).

Almácigo colorado o amarillo. Bursera simaruba L. (burceráceas). Roig especifica que, según Mazá, la coloración de la especie depende probablemente de la calidad del terreno.
\end{abstract}

Almaje de Santo Domingo. Errata evidente, se trata de mameyes de Santo Domingo.

Anón. Annona squamosa L. (anonáceas). Parra no describe en detalle la especie. Hay otras variedades en Cuba como A. cubensis R. E. Fries; $A$. Bullata, A. Rich; A. habanensis R. E. Fries; A. lutescens Safford.

Ateje común. Cordia cocolloca L. (boragináceas). La descripción de Parra coincide más con esta especie que con el ateje hembra (C. valenzuelana Rich.), anotada por Barras de Aragón.

Caña brava. Bambusa vulgaris Schard. (B. arundinacea Willd) (gramináceas). Por la descripción de Parra no puede ser Gynerium saccharoides, que es más pequeña y fina.

Castaño purgante de Cuba (lirio francés). Pristimera wrigtii Ness. (Hippocratea malpighiafolia, Rudge) (celastráceas). Según Maza y Ferrer - dice Roig - también se le da ese nombre a la Jatropha multifida L. (euforbiáceas).

Cedro. Cedrela mexicana M. J. Roem. (meliáceas). Más común en Cuba. Según Roig, $C$. odorata $\mathrm{L}$. no es cubana, aunque existe en alguna parte de 


\section{LA OBRA BOTÁNICA DE ANTONIO PARRA}

Cuba como Isla de Pinos. Parece ser el cedro caoba. Es común a Jamaica y Puerto Rico.

Ceiba. Ceiba pentandra L. (bombáceas). Es la única especie en Cuba, según Roig. Eriodendron aesculifolium D. C., es una especie hermana introducida en México.

Cerillo. Exostema caribaeum (Jacq.) Roen y Schult. (rubiáceas). Otra especies, llamadas cerillos, pudieran ser también las enviadas por Parra, o sea Rochefortia grandiflora Ekman (boragináceas) y Schoephia chrysophylloides (A. Rich) Planc. (olacáceas).

Cornezuelo. Cuernecillo. Acacia spadicigera Cham y Schul. (A. cornigera Willd) (leguminosas).

Frijol anbril. La descripción de Parra es muy pobre y la variedad de frijoles es grande en Cuba. Pudiera ser, por las flores, Phaseolus calculatus Roxb. (leguminosas).

Guacamaya colorada. Poinciana pulcherrima, L. (cesalpináceas).

Guacamaya amarilla (de laguna). Cassia aculiata Pahl. (cesalpináceas). También tiene flores amarillas la llamada francesa (C. alata L.).

Guacima. Guazuma tomentosa H. B. K. (esterculiáceas).

Guayacamaya. (sic) Ver guacamaya.

Gomitil blanco o Gomitel. (sic) Ver Vomitel.

Guayabita del Pinar. Psidium guayabita A. Rich. (mirtáceas).

Guayabo. Psidium guajaba L. (mirtáceas).

Guayaba del Perú. Psidium guajaba L. (mirtáceas).

Guira chica. (cimarrona). Crescentia cujete L. (bignonáceas), por la descripción de Parra. Es una variedad de la grande.

Guira grande. Crescentia cujete L. (bignon áceas).

Jagua. Genipa americana L. (rubiáceas).

Jobo. Spondias mombin L. (anacardiáceas). Según Roig es la única especie que existe en Cuba.

Jolio. (sic) Ver Jobo. 


\section{ARMANDO GARCíA GONZÁLEZ}

Lirio francés. Ver Castaño purgante.

Mamey colorado. Calocarpon sapota (Jaq.) Merr. (Lucuma mamosa A. D. C.) (sapotáceas).

Mamey de Santo Domingo. Mammea americana L. (clusiáceas).

Mamón. Annona reticulata L. (anonáceas).

Mamoncillo. Mellicocca bijuga L. (sapindáceas).

Manzanillo. Hippomane mancinella L. (euforbiáceas).

Marañón. Anacardium occidentale L. (anacardiáceas).

Palma de cocos. Cocos nucifera L. (palmáceas).

Palma real de Cuba. Roystonea regia H. B. K. (palmáceas).

Papaya. Carica papaya L. (caricáceas).

Piñón botija. Jatropha curcas L. (euforbiáceas).

Piñón espinoso. Erythrina cubensis C. Wr. (papilionáceas).

Tamarindo. Tamarindus indica L. (cesalpináceas).

Tobo. (sic) Ver Jobo.

Toba. (sic) Ver Jobo.

Uvero de Cuba. (uva caleta). Coccoloba uvifera Jacq. (poligonáceas).

Vomitel blanco. Ateje blanco. Cordia galeottiana A. Rich. (boragináceas). Por la descripción de Parra parece ser esta especie.

Zapote. Manilkara zapodilla (Jacq.) Gilly (Achras sapota L.) (sapotáceas).

Zapote culebra. Pouteria domingensis (Gris.) Bachai. (sapotáceas).

Zapote negro. Dyospiros laurifolia A. Rich. (sapotáceas). 


\begin{abstract}
APENDICE II
Asimismo creemos oportuno reproducir —respetando la ortografía, salvo erratas de impresión evidentes- la clasificación efectuada por Barras de Aragón acerca de las plantas envíadas por Parra, para lo cual se valió, como aquél mismo afirma, del Diccionario de nombres vulgares de muchas plantas, de Miguel Colmeiro, así como del Diccionario Enciclopédico Espasa. Barras expresa que su lista es mayor que la de Parra porque muchos de los de esta naturaleza tienen nombre genérico, y en esos casos él (Barras) escribió las especies cubanas correspondientes. De todo ello resulta, según su parecer, que Parra envió a España cincuenta especies de plantas de Cuba.
\end{abstract}

Abrojo de la Florida. Pereskia portulacaefolia Haw. (cactáceas).

Aeje (sic, Ateje) hembra de Cuba. Cordia valenzuelana Rich. (boragináceas).

Aguacate. Persea gratissima Gartn. (lauráceas).

Almácigo amarillo de Cuba. Bursera gumnifera Jacq. (terebintáceas).

Almaje de Santo Domingo.

Anona de las Antillas. Anonna muricata L. (anonáceas).

Anones. Annona obtusifolia Tuss., propia de las Antillas (anonáceas).

Annona squamosa L. Originaria del Perú y Colombia, cultivada en los paises tropicales (anonáceas).

Atexe-Ateje blanco de Cuba. Cordia alba Roen. et Schultz (borragináceas).

Atexe de Cuba. Cordia collococa L. (boragináceas).

Caimito. Caimitillo de las Antillas. Coryophylum olivaeforme Lam. et C. microphylum D. C., etc. (sapotáceas).

Caimito de las Antillas. Chrysophylum caimito L. (sapotáceas).

Castaño purgante de Cuba. Hippocratia malyghifolia Rudi. (hipocastáneas).

Asclepio-Vol. XLVII-2-1995 
Caña brava de Cunmana. Gynerium saccharoides B. et Kuntz. (gramináceas).

Caña brava del Orinoco. Gradua atifolia Kunth. (gramináceas).

Cedro de las Antillas y Barbadas. Cedrela adorata L. (meliáceas).

Ceiba de Cuba. Eriodendron anfroctuosum D. C. (bombáceas).

Cerillo. Symphonia dolulifera L. (hipericáceas).

Cuernecillo de Cuba. Acacia cornigera Willd (leguminosas).

Cuernos de Veracruz?

Frijol?

Frijol Antillano?

Frijol caballero de Cuba. Lablau vulgaris Lav. (leguminosas).

Frijol cimarrón de Cuba. Vicia vexilata Rich. et luteola Berth (leguminosas).

Frijol de olor de la Habana. Clitoria speciosa Cav. (leguminosas).

Guayaco. Guayacum sanctum L., Guayacum verticale Ortega.

Guacamaya colorada.

Guacamaya de costa. Cesalpinia bijuga Sw. (leguminosas).

Guacamaya de Cuba. Poinciana pulcherrima L. (leguminosas).

Guacamaya francesa de Cuba. Cassia alata L. (leguminosas).

Guacima amarilla o Guacima varia de Cuba. Luhea platypetala Rich. (tiliáceas).

Guanabano de loma o cimarrón de Cuba. Annona montana Maef. (anonáceas).

Gomitil blanco?

Guayabitos del pinar de Cuba. Psidium guayabita Rich. (mirtáceas).

Guayabo. Psidium guayaba (mirtáceas).

Guayabo agrio de las Antillas. Psidium pomiferium. Es el mismo cotorrero y silvestre de Cuba (mirtáceas).

Guayaba del Perú. Psidium pyriferum. Es el mismo blanco de Cuba (mirtáceas).

Guayacamaya? 


\section{LA OBRA BOTÁNICA DE ANTONIO PARRA}

Guira cimarrona de Cuba. Crescentia acuminata H. B. et Kunth. (bignoniáceas).

Guira criolla de Cuba. Crescentia cucurbitina L. (bignoniáceas).

Guiras chicas? ¿Es el Guirito de Cuba?. Solanum mammosum L. (solanáceas).

Jagua de Cuba. Genipa americana L. (rubiáceas). Jolio?

Lirio francés de Cuba, que otros llaman castaño, y su fruto es purgante.

Mamey de las Antillas. Mammea americana L. (gutíferas).

Mamey colorado de Cuba. Lucuma mammosa Gaesrn et L. Bomplandi $\mathrm{H}$. B. et Kunth. (sapotáceas).

Mamón de Cuba. Annona reticulata L. (anonáceas).

Momoncillo de Cuba. Melicoa bijuga L. Es el mismo mamón de Caracas (sapindáceas).

Manzanillo de Cuba. Hippomene mancinella L. (euforbiáceas).

Palma de cocos. Cocos nucifera L. (palmáceas).

Palma Real de Cuba. Oreodoxia regia H. B. et Kunth. (palmáceas).

Papaya o Papayo de Cuba. Carica papaya L. (papayáceas).

Piñón botija o purgante de Cuba. Curcas purgans (euforbiáceas).

Piñón espinoso de las Antillas. Erytryna coralledendron L. (leguminosas).

Tamarindo de America. Tamarindus occidentalis Gaertn (leguminosas).

Tobo?

Toba. Onopordon acanthium L. (compuestas).

Uvero de Cuba o uvas caleta de Cuba. Coceoloba uvifera L. (poligonáceas). Zapote de las Antillas o Zapote pequeño. Sapota achras Mill. (sapotáceas). Zapote de Culebra de Cuba. Lucuma serpentaria H. B. et Kunth (sapotáceas).

Zapote negro de Cuba. Diospyros laurifolia Rich. (ebanáceas). 